\title{
Dissecting myocardial signal transduction cascades: Sharp new insights from multi-tracer molecular imaging
}

\author{
Frank M. Bengel, MD
}

\section{See related article, pp. 254-263}

The potential to combine tracers for interrogation of several components of disease within a single imaging session has always been a strength of nuclear imaging. In conventional scintigraphy and SPECT, this can be achieved by sequential or simultaneous imaging of two isotopes with different photon energies. Examples are the combination of thallium-201 and Tc-99m-labeled agents for rapid assessment of myocardial perfusion at rest and stress, ${ }^{1,2}$ or the combination of iodine-123- or indium-111-labeled molecular-targeted tracers with a perfusion imaging agent. ${ }^{3,4}$ In PET, this is achieved by sequential imaging of two or more short-lived compounds. Examples are the combined assessment of myocardial perfusion and glucose utilization for determination of myocardial viability, ${ }^{5}$ or the combination of multiple radiolabeled substrates for detailed investigation of cardiac metabolism. ${ }^{6}$

The advent of molecular imaging results in an increasing variety of increasingly specific tracers. ${ }^{7,8}$ When used alone, these tracers provide a snapshot of the presence or absence of abnormality of the interrogated molecular target structure. This snapshot may be difficult to interpret, because the role of upstream or downstream events along the signal transduction cascade remains obscure. Combination of multiple tracers, which target a series of different molecules along a signal transduction cascade, would thus be attractive. Dissection of the cascade would not only allow for

From the Division of Nuclear Medicine, Russell H. Morgan Department of Radiology and Radiological Science, Johns Hopkins University, Baltimore, MD.

Reprint requests: Frank M. Bengel, MD, Division of Nuclear Medicine, Russell H. Morgan Department of Radiology and Radiological Science, Johns Hopkins University, $601 \mathrm{~N}$. Caroline Street/JHOC 3225, Baltimore, MD 21287; fbengel1@jhmi.edu.

J Nucl Cardiol 2010;17:175-6.

$1071-3581 / \$ 34.00$

Copyright $(2010$ by the American Society of Nuclear Cardiology. doi:10.1007/s12350-010-9202-x improved understanding of pathogenetic mechanisms, it may also allow for identification of the most relevant molecular target structure, which is most severely affected by the disease. This structure may then serve as a single target in subsequent efforts to simplify imaging for broader clinical application.

The study by Kenk et al in this issue of the Journal of Nuclear Cardiology is an elegant and innovative step into this direction. ${ }^{9}$ The authors chose the sympathetic nervous system, which is of increasing interest to the cardiovascular imaging community due to its key role in regulation of contractility and electromechanic properties in health and disease. ${ }^{10-12}$ They combined three different tracers to dissect noradrenergic signaling in a rodent model of anthracycline-induced cardiotoxicity. For this purpose, they did not only use a radiolabeled catecholamine analogue to look at presynaptic nerve terminal integrity and a radiolabeled beta-adrenergic receptor ligand to look at postsynaptic receptor density-an approach that had been pursued by others before. ${ }^{13-15}$ Importantly, the authors added a marker of downstream signaling to their mix, namely the phosphodiesterase inhibitor carbon-11 rolipram which is involved in regulation of cyclic AMP. In brief, the authors found no significant change in presynaptic catecholamine uptake and downstream phosphodiesterase binding, but they observed a significant reduction in postsynaptic beta-receptor density after 3 weeks of treatment with anthracycline, at a time where cardiac function was still preserved. On the one hand, their results suggest that, if a single tracer is sought for early clinical detection of cardiotoxicity, beta-adrenergic receptor imaging may be the best choice. On the other hand, the observation that upstream presynaptic catecholamine uptake as well as downstream cyclic AMP regulation can remain unchanged despite changes in receptor density as the intermediary structure in the signaling cascade is new and needs to be clarified in further mechanistic studies.

Some limitations of the work by Kenk et al need to be recognized. Firstly, the study was done using autoradiography, not in vivo imaging. Autoradiography allows for a robust, high-resolution assessment of myocardial radioactivity, but it is a post-mortem ex vivo 
technique and eliminates factors such as bloodpool activity, partial volume effects, and metabolites which are critical to the feasibility of in vivo imaging. Secondly, myocardial blood flow was not determined. Myocardial retention of the employed tracers may be flow-dependent, and differences in flow-dependency of those tracers may explain differences in their ability to detect cardiotoxicity. And finally, the rat was used as a model but the sympathetic nervous system in these small rodents may differ from that in larger mammals and humans. ${ }^{16}$ More work will be necessary to determine the feasibility and robustness of this multi-tracer approach for in vivo imaging and to translate it into a clinical setting.

Despite these limitations, the study by Kenk et al should still be seen as highly innovative and stimulating work, which represents an important early step into the right direction: In times of an ever increasing specificity of molecular medicine, the combination of multiple probes will provide deeper insights into molecular pathways and will help to further establish the value of noninvasive targeted imaging. It is with this work like with most innovative projects: Further questions arise, and a stimulus for subsequent efforts is provided. Based on the results of this study, it is likely that we will see additional investigations into the pathobiology of noradrenergic signaling, further efforts to establish in vivo imaging methodology, and further work to translate the knowledge into a clinical setting in the future.

\section{References}

1. Berman DS, Kang X, Tamarappoo B, Wolak A, Hayes SW, Nakazato R, et al. Stress thallium-201/rest technetium-99m sequential dual isotope high-speed myocardial perfusion imaging. JACC Cardiovasc Imaging 2009;2:273-82.

2. Kadrmas DJ, Frey EC, Tsui BM. Simultaneous technetium-99m/ thallium-201 SPECT imaging with model-based compensation for cross-contaminating effects. Phys Med Biol 1999;44:1843-60.

3. Matsunari I, Schricke U, Bengel FM, Haase HU, Barthel P, Schmidt G, et al. Extent of cardiac sympathetic neuronal damage is determined by the area of ischemia in patients with acute coronary syndromes. Circulation 2000;101:2579-85.

4. Meoli DF, Sadeghi MM, Krassilnikova S, Bourke BN, Giordano FJ, Dione DP, et al. Noninvasive imaging of myocardial angiogenesis following experimental myocardial infarction. J Clin Invest 2004;113:1684-91.

5. Tillisch J, Brunken R, Marshall R, Schwaiger M, Mandelkern M, Phelps M, et al. Reversibility of cardiac wall-motion abnormalities predicted by positron tomography. N Engl J Med 1986;314:884-8.

6. Peterson LR, Herrero P, Schechtman KB, Racette SB, Waggoner $\mathrm{AD}$, Kisrieva-Ware Z, et al. Effect of obesity and insulin resistance on myocardial substrate metabolism and efficiency in young women. Circulation 2004;109:2191-6.

7. Nahrendorf M, Sosnovik DE, French BA, Swirski FK, Bengel F, Sadeghi MM, et al. Multimodality cardiovascular molecular imaging, part II. Circ Cardiovasc Imaging 2009;2:56-70.

8. Sinusas AJ, Bengel F, Nahrendorf M, Epstein FH, Wu JC, Villanueva FS, et al. Multimodality cardiovascular molecular imaging, part I. Circ Cardiovasc Imaging 2008;1:244-56.

9. Kenk M, Thackeray JT, Thorn SL, Dhami K, Chow BJ, Ascah KJ, et al. Alterations of pre- and post-synaptic noradrenergic signaling in a rat model of adriamycin-induced cardiotoxicity. J Nucl Cardiol 2010. doi:10.1007/s12350-009-9190-X.

10. Bengel FM. Clinical cardiovascular molecular imaging. J Nucl Med 2009;50:837-40.

11. Bengel FM, Schwaiger M. Assessment of cardiac sympathetic neuronal function using PET imaging. J Nucl Cardiol 2004;11: 603-16.

12. Sasano T, Abraham MR, Chang KC, Ashikaga H, Mills KJ, Holt $\mathrm{DP}$, et al. Abnormal sympathetic innervation of viable myocardium and the substrate of ventricular tachycardia after myocardial infarction. J Am Coll Cardiol 2008;51:2266-75.

13. Caldwell JH, Link JM, Levy WC, Poole JE, Stratton JR. Evidence for pre- to postsynaptic mismatch of the cardiac sympathetic nervous system in ischemic congestive heart failure. J Nucl Med 2008;49:234-41.

14. Schafers M, Dutka D, Rhodes CG, Lammertsma AA, Hermansen F, Schober O, et al. Myocardial presynaptic and postsynaptic autonomic dysfunction in hypertrophic cardiomyopathy. Circ Res 1998;82:57-62.

15. Tsukamoto T, Morita K, Naya M, Inubushi M, Katoh C, Nishijima $\mathrm{K}$, et al. Decreased myocardial beta-adrenergic receptor density in relation to increased sympathetic tone in patients with nonischemic cardiomyopathy. J Nucl Med 2007;48:1777-82.

16. Tipre DN, Fox JJ, Holt DP, Green G, Yu J, Pomper M, et al. In vivo PET imaging of cardiac presynaptic sympathoneuronal mechanisms in the rat. J Nucl Med 2008;49:1189-95. 